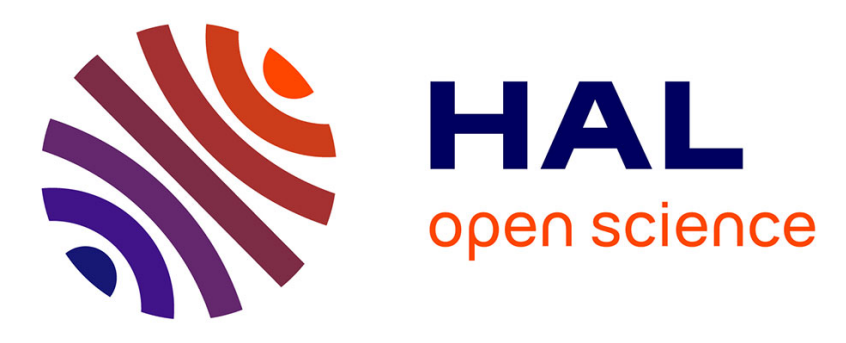

\title{
Venous thrombosis supervised image indexing and fuzzy retrieval
}

\author{
Anas Dahabiah, John Puentes, Basel Solaiman
}

\section{To cite this version:}

Anas Dahabiah, John Puentes, Basel Solaiman. Venous thrombosis supervised image indexing and fuzzy retrieval. EMBS 2007: 29th annual international conference of the IEEE engineering in medicine and biology society, August 22-26, Lyon, France, Aug 2007, Lyon, France. pp.4528 - 4531, 10.1109/IEMBS.2007.4353346 . hal-02121490

\section{HAL Id: hal-02121490 \\ https://hal.science/hal-02121490}

Submitted on 9 Jun 2021

HAL is a multi-disciplinary open access archive for the deposit and dissemination of scientific research documents, whether they are published or not. The documents may come from teaching and research institutions in France or abroad, or from public or private research centers.
L'archive ouverte pluridisciplinaire HAL, est destinée au dépôt et à la diffusion de documents scientifiques de niveau recherche, publiés ou non, émanant des établissements d'enseignement et de recherche français ou étrangers, des laboratoires publics ou privés. 


\title{
Venous Thrombosis Supervised Image Indexing and Fuzzy Retrieval
}

\author{
A. Dahabiah, J. Puentes, and B. Solaiman \\ ENST Bretagne, GET-ENST Département Image et Traitement de l'Information, Brest, France \\ INSERM, U650, Laboratoire de Traitement de l'Information Médicale, Brest, France
}

\begin{abstract}
Clinical assessment of venous thrombosis (VT) is essential to evaluate the risk of size increase or embolism. Analyses like echogenecity and echostructure characterization, examine ancillary evidence to improve diagnosis. However, such analyses are inherently uncertain and operator dependent, adding enormous complexity to the task of indexing diagnosed images for medical practice support, by retrieving similar images, or to exploit electronic patient record repositories for data mining. This paper proposes a VT ultrasound image indexing and retrieval approach, which shows the suitability of neural network VT characterization, combined with a fuzzy similarity. Three types of image descriptors (sliding window, wavelet coefficients energy and co-occurrence matrix), are processed by three different neural networks, producing equivalent VT characterizations. Resulting values are projected on fuzzy membership functions and then compared with the fuzzy similarity. Compared to nominal and Euclidean distances, an experimental validation indicates that the fuzzy similarity increases image retrieval precision beyond the identification of images that belong to the same diagnostic class, taking into account the characterization result uncertainty, and allowing the user to privilege any particular feature.
\end{abstract}

Keywords - Similarity, venous thrombosis, supervised image indexing, fuzzy retrieval, echogenicity, echostructure, neural network classifier, and ultrasound.

\section{INTRODUCTION}

Venous thrombosis (VT) is a blood clot most commonly formed in the legs, or other parts of the body, that interferes with circulation producing inflammation of the affected vein. It can be superficial or deep, having the potential of being life threatening, if it breaks loose traveling through the blood stream, causing severe damage, principally to the lungs, heart, and brain. Therefore, VT clinical assessment is essential to evaluate the risk of size increase or embolism. Ultrasound (US) is a specialist's choice to track the VT along the veins of the lower extremities up to the abdomen, neck and arms, because it is non-invasive, rapid and of relative low cost. US images permit a qualitative evaluation of the VT aspect, but analyses like echogenecity and echostructure characterization, among others, are necessary to obtain ancillary evidence that could improve diagnosis [1-3], despite their inherent uncertainty and operator dependence.

VT echogenecity is characterized as anechogeneous, hypoechogeneous, isoechogeneous, or hyperechogeneous, while echostructure can be qualified as homogeneous, heterogeneous, or very heterogeneous. Such VT characterization, adds enormous complexity to the task of indexing diagnosed images for medical practice support by retrieving similar images, or to exploit electronic patient record repositories for data mining. Known approaches to index and retrieve images, identify most of those that belong to the same diagnostic class depending on a pre-defined threshold criterion, without appraising the similarity accuracy degree [4]. There is currently no method described specifically to increase image retrieval precision, considering the characterization uncertainty. Our approach proposes to flexibly exploit VT characterization uncertainty and retrieve the most similar images within a diagnosis class. Applying a fuzzy similarity, it allows outlining the importance of either the characterization parameters, or the complementary elements that describe the case.

Using a standard image indexing and retrieval system architecture (section II), three types of image descriptors (sliding window, wavelet coefficients energy and cooccurrence matrix) are processed by three neural networks to generate equivalent echogenecity and echostructure characterizations (section III). The fuzzy similarity measure is then defined (section IV) and experimentally validated using a detailed example (section V), before discussing the results and stating the conclusion (section VI).

\section{INDEXING AND RETRIEVAL SYSTEM}

Image indexing and retrieval are carried out in two main steps (Fig. 1): first, images that belong to the database are processed off-line, extracting the image descriptors and characterizing the VTs to create the image signatures metadata database; then, the query image is processed on-line in the same manner and its characterized features compared to the ones available in the meta-data database, by means of a fuzzy similarity measure, to retrieve the similar images.

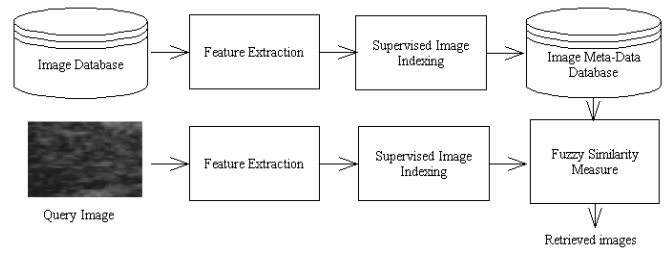

Fig. 1. Image indexing and retrieval system.

Commonly, image signatures are directly calculated by extracting significant visual features or transformed values, and the distance between them is measured to identify those that belong to the query image class. Our approach processes 
image descriptors using three neural networks that characterize VTs in an equivalent manner. The obtained values are projected on fuzzy membership functions to assess their degree of truth, and compared using a fuzzy similarity, to retrieve similar images in the sense of VT echogenecity and echostructure.

\section{VT FEATURE EXTRACTION AND CHARACTERIZATION}

An image feature vector of minimal size and capable of describing each type of VT in a unique way is first calculated. Thereafter, depending on the extracted features, an adapted feed forward artificial neural network (ANN) that makes use of a global (by epoch) and supervised learning [5], generates the respective echogenecity and echostructure image characterization labels. A brief description of the three most performing feature vector - ANN association is presented next. Experimental results show that all of them provide equivalent image characterization (see [2] for details on feature vectors, ANN performance and VT description).

\section{A. Sliding window descriptor}

The VT analysis zone is defined with a 30x30 pixel region of interest (ROI), which is swept by a $3 \times 3$ averaging window. Each calculated average is normalized to provide a 100 value vector used as input. This feature vector is processed by a two layer ANN in which the activation function of the hidden layer is the sigmoid, and that of the output layer is the linear function (Fig. 2).

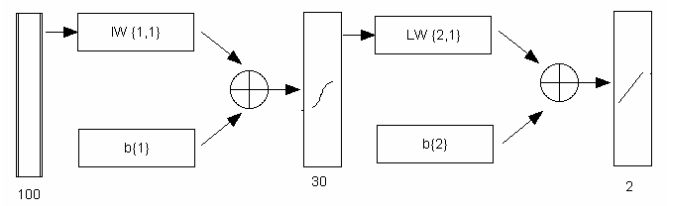

Fig. 2. ANN architecture to process the sliding window output ('b' is the bias, 'IW' are the input weights, and 'LW' are the layer weights).

\section{B. Wavelet coefficients energy descriptor}

The VT ROI is decomposed in four spatial frequency subbands, at six consecutive levels applying biorthonormal wavelets, to assure that the feature vector is invariant to translations [6]. Energy calculation of the resulting subbands coefficients generates 24 feature vector elements. This feature vector is processed by a simple ANN without a hidden layer, using Levenberg Marquardt learning algorithm [7], activated by a linear function (Fig. 3).

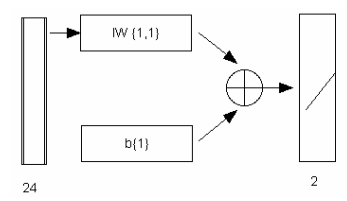

Fig. 3. ANN architecture to process the wavelet-based descriptor.

\section{Co-occurrence matrix descriptor}

VT co-occurrence matrices are calculated in four directions $\left(0^{\circ}, 45^{\circ}, 90^{\circ}, 135^{\circ}\right)$ and four distances $(1,2,3,4$ pixels) between neighboring pixels. Subsequently, for each of the 16 matrices, energy, entropy, inertia, and homogeneity are calculated to produce 64 distinct elements. This feature vector is processed by a two-layer ANN, identical to the one used to characterize the sliding window descriptor (Fig. 4).

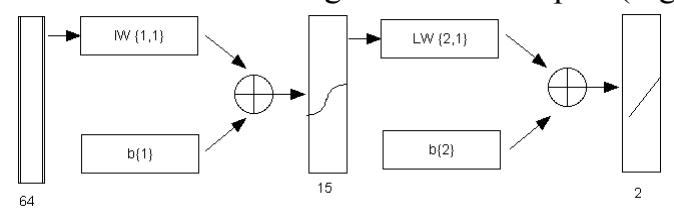

Fig. 4. ANN architecture to process the co-occurrence matrix descriptor.

\section{VT IMAGE SIMILARITY MEASURE}

As explained in section I, VT echogenicity can be described using four labels, and VT echostructure using three labels. The previously described ANNs are capable of recognizing both characteristics of a given VT image unambiguously, by rounding the output to the nearest learned value. This result allows identifying most of the images that belong to the same diagnosis class, excluding simultaneously the possibility to exploit the obtained characterization uncertainty, and to improve retrieval precision.

Our work proposes therefore, to take advantage of the membership degree to each characteristic label, fuzzifying the ANNs output by means of a membership function. Accordingly, the related similarity measure should estimate the proximity between two vectors, independently described by their element membership degrees to their respective multi-label characterization. Furthermore, in a data mining context, it would be necessary to weight the relative importance of the obtained image characteristic labels, particularly when the relation between similar electronic patient records is examined. This implies that label weights should also be accounted by the similarity measure. Lastly, to achieve a precise retrieval, which is very important in data mining rule generation, it is required to integrate the characterization labels certainty and accuracy. For instance, if the echogenicity certainty is higher than that of echostructure, because the image descriptors are considered as more accurate than those describing the echostructure, the echogenicity weight will be proportionally higher than the echostructure weight. The fuzzy similarity measure stated by Zhang [8] satisfies to a large extent the aforementioned requirements.

\section{A. Fuzzy semantic distance}

Assuming that a multimedia object is described by a set of $N$ features $\left\{f_{1}, f_{2}, \ldots, f_{N}\right\}$, and that each feature $f i$ can be characterized by fuzzy subsets $\left\{A_{i 1}, A_{i 2}, \ldots, A_{i p}\right\}$, it is possible to define a fuzzy space in which $\mu_{A_{i j}}(f i)$ represents the membership degree of feature $f_{i}$ within it. For this case, the feature inclusion in the corresponding fuzzy space is directly proportional to the membership degree. Hence, the fuzzy feature vector description $(F)$ of an object $O$ is defined as: 


$$
F=\left\{\begin{array}{cccc}
\mu_{11}(f 1) & \mu_{12}(f 1) & \ldots \ldots & \mu_{1 m(1)}(f 1) \\
\mu_{21}(f 2) & \mu_{22}(f 2) & \ldots \ldots & \mu_{2 m(2)}(f 2) \\
\ldots \ldots & \ldots \ldots & \ldots \ldots & \ldots \ldots \\
\mu_{N 1}(f N) & \mu_{N 2}(f N) & \ldots \ldots & \mu_{N m(n)}(f N)
\end{array}\right.
$$

Where $m(i)$ represents the number of fuzzy sets, necessary to describe feature $f_{i}$. At a practical level, object retrieval entails evaluating the similarity degree between a query object and the available objects in the database. Assuming that two multimedia objects $O_{1}$ and $O_{2}$, are represented by fuzzy feature vectors using (1), the fuzzy semantic distance $(S D)$ between them is defined as follows:

$$
S D\left(O_{1}, O_{2}\right)=\sum_{i=1}^{N}\left(w_{i} * \sum_{j=1}^{m(i)} w_{i j} *\left|\mu_{i j}\left(O_{1}\right)-\mu_{i j}\left(O_{2}\right)\right|\right)
$$

Where $\mu_{i j}$ is the membership function, and $w_{i}$ and $w_{i j}$ are the characterization uncertainty weights, that take different values according to the defined retrieval requirements. These three parameters must satisfy the following conditions:

$$
\sum_{j=1}^{m(i)} w_{i j}=1, \sum_{j=1}^{m(i)} \mu_{i j}=1, \sum_{i=1}^{N} w_{i}=1
$$

\section{B. Fuzzy similarity}

Applying the previously defined fuzzy semantic distance (2), the fuzzy similarity $\left(\operatorname{Sim}\left(O_{1}, O_{2}\right)\right)$ measure between two fuzzy subsets can be calculated according to:

$$
\operatorname{Sim}\left(O_{1}, O_{2}\right)=\frac{\sum_{i=1}^{N}\left(w_{i} * \sum_{j=1}^{m(i)} w_{i j} * \operatorname{Min}\left(\mu_{i j}\left(O_{1}\right), \mu_{i j}\left(O_{2}\right)\right)\right)}{\sum_{i=1}^{N}\left(w_{i} * \sum_{j=1}^{m(i)} w_{i j} * \operatorname{Max}\left(\mu_{i j}\left(O_{1}\right), \mu_{i j}\left(O_{2}\right)\right)\right)}
$$

Here, $w_{i}$ weights the importance of feature $f_{i}$ compared to the others, and $w_{i j}$ enables to assign the importance of each subfeature related to the main feature $f_{i}$, compared to the other sub-features.

\section{EXPERIMENTAL VALIDATION}

To demonstrate the image retrieval precision increase obtained with the proposed approach, a simple experiment has been designed. Given a VT image database $(\Omega)$, which for the sake of clarity and to show the proposed approach interest, only has five images: $\Omega=\left\{I_{1}, I_{2}, \ldots I_{5}\right\}$, each image echogenicity and echostructure are characterized using any of the three methods described in section III. Additionally, both the medical specialists and the characterization algorithms have labeled the 5 images as follows (Fig. 5): $\mathrm{I}_{1}$, $\mathrm{I}_{2}, \quad \mathrm{I}_{5}$ are isoechogeneous and homogeneous; $\mathrm{I}_{3}$ is hypoechogeneous and homogeneous; $\mathrm{I}_{4}$ is isoechogeneous and heterogeneous. A query image I, evaluated also by the medical specialist and the characterization algorithms, as isoechogeneous and homogeneous, is used to compare the obtained fuzzy similarity retrieval results, to nominal and Euclidean distances retrieval.

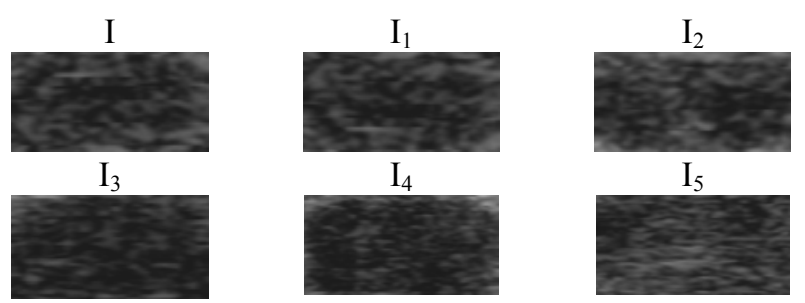

Fig. 5. VT query image (I) and $\Omega$ database images $\left(I_{1}, I_{2}, I_{3}, I_{4}, I_{5}\right)$.

\section{A. Nominal distance}

When the nominal distance measure is applied, each subfeature provided by the ANN is coded with two binary variables. For instance, echogenicity labels are coded in the following manner: hypoechogenicity $(0,0)$, hyperechogenicity $(1,0)$, isoechogenicity $(0,1)$, and anechogenicity $(1,1)$. Echostructure labels are coded as: homogeneity $(0,0)$, heterogeneity $(0,1)$, and hyperheterogeneity $(1,0)$. The same coding scheme is used because features are independently evaluated, without cross measurements between fuzzy sub-sets. Thereafter, the distance $d_{I_{i}, I_{j}}$ between I and images in $\Omega$ is:

$$
\begin{aligned}
& d_{I, I_{1}}=d_{I, I_{2}}=d_{I, I_{5}}=0 \\
& d_{I, I_{3}}=0.5[0.5(0+1)+0.5(0+0)]=0.25 \\
& d_{I, I_{4}}=0.5[0.5(0+0)+0.5(0+1)]=0.25
\end{aligned}
$$

Results indicate as expected, that $\mathrm{I}_{1}, \mathrm{I}_{2}, \mathrm{I}_{5}$, are identical to I. Conversely, $\mathrm{I}_{3}$ and $\mathrm{I}_{4}$ are equally similar to $\mathrm{I}$, even if they are rather different. Note that any other coding will give equivalent results, but with different values.

\section{B. Euclidean distance}

To retrieve similar images using the Euclidean distance, it is only necessary to calculate the feature vector, for instance, the co-occurrence-matrix-descriptors. Applied to $\Omega$ the following results are obtained:

$$
\left.\begin{array}{l}
d_{V_{I}, V_{I_{1}}}=d_{V_{I}, V_{I_{2}}}=212.22 ; \quad d_{V_{I}, V_{I_{3}}}=287.84 \\
d_{V_{I}, V_{I_{4}}}=701.22 ; \quad d_{V_{I}, V_{I_{5}}}=217.66
\end{array}\right\}
$$

Retrieved images can be represented as $\left\{\left(\mathrm{I}_{1}, \mathrm{I}_{2}\right), \mathrm{I}_{5}, \mathrm{I}_{3}, \mathrm{I}_{4}\right\}$, to indicate that $I_{1}$ and $I_{2}$ are the most similar images to $I$, with the same relative importance. Additionally, $\mathrm{I}_{5}$ is less similar to I than $\left(\mathrm{I}_{1}, \mathrm{I}_{2}\right)$ and so on. Compared to the nominal distance, the Euclidean distance is more precise since it distinguishes $\left(I_{1}, I_{2}\right)$ from $I_{5}$. Note that obtaining two identical distances does not imply that the related feature vectors are the same.

\section{Fuzzy similarity}

A membership function like the triangular function is applied to fuzzify the ANN output. In this way, the measurement of each image $I_{i}$ membership degree to the echogenicity labels, becomes a four-element membership degree vector $\left(\mu_{\text {hypo }, I_{i}}, \mu_{i s o, I_{i}}, \mu_{\text {hyper }, I_{i}}, \mu_{\text {anecho }, I_{i}}\right)$, and the membership degree to echostructure labels a three-element 
vector $\left(\mu_{\text {homogeneous }, I_{i}}, \mu_{\text {heterogeneous }, I_{i}}, \mu_{\text {veryhetro } I_{i}}\right)$. Each one of $\Omega$ images is consequently represented as:

$$
\left.\begin{array}{rl}
I_{1} & \mapsto[(0.12,0.88,0,0),(0.70,0.30,0)] \\
I_{2} & \mapsto[(0.18,0.82,0,0),(0.65,0.35,0)] \\
I_{3} & \mapsto[(0.65,0.35,0,0),(0.70,0.30,0)] \\
I_{4} & \mapsto[(0.35,0.65,0,0),(0.35,0.65,0)] \\
I_{5} & \mapsto[(0.30,0.70,0,0),(0.55,0.45,0)]
\end{array}\right\}
$$

And the query image $I$ is represented by:

$$
I \mapsto[(0.10,0.90,0,0),(0.70,0.30,0)]
$$

Assuming that the echogenicity and echostructure have the same importance $\left(w_{l}=w_{2}=0.5\right)$, the fuzzy similarity gives the following results.

$$
\begin{aligned}
& d_{I, I_{1}}=0.005 ; \quad d_{I, I_{2}}=0.03667 ; \quad d_{I, I_{3}}=0.1375 \\
& d_{I, I_{4}}=0.179167 ; \quad d_{I, I_{5}}=0.1
\end{aligned}
$$

Ordering them from the most to the least similar image, we obtain: $\left\{I_{1}, I_{2}, I_{5}, I_{3}, I_{4}\right\}$. Despite the similarity of this sequence with the output obtained using the Euclidean distance, this result is more precise because it can strictly distinguish the images, particularly $\mathrm{I}_{1}$ and $\mathrm{I}_{2}$, considering that $\mathrm{I}_{1}$ is more similar to $\mathrm{I}$ than $\mathrm{I}_{2}$, when the small differences between adjacent sub-labels are taken into account.

On the contrary, the user could be more interested in echostructure than echogenicity similarity (e.g. $w_{l}=0.25$, $w_{2}=0.75$ ). In this case, the fuzzy similarity provides different results:

$$
\begin{aligned}
& d_{I, I_{1}}=0.0025 \\
& d_{I, I_{2}}=0.035 ; \quad d_{I, I_{3}}=0.06875 \\
& d_{I, I_{4}}=0.20625 ; \quad d_{I, I_{5}}=0.1
\end{aligned}
$$

As a consequence, the relevance order of the retrieved images is: $\left\{\mathrm{I}_{1}, \mathrm{I}_{2}, \mathrm{I}_{3}, \mathrm{I}_{5}, \mathrm{I}_{4}\right\}$, which is a very interesting outcome. Although $\mathrm{I}_{1}, \mathrm{I}_{2}, \mathrm{I}_{5}$ have the same characteristics of I (isoechogeneous, homogeneous), $\mathrm{I}_{3}$ whose echogenicity is different from the measured value on $\mathrm{I}$, is identified as being more similar to $\mathrm{I}$ than $\mathrm{I}_{5}$, given that its echostructure (the significant characteristic for the user), matches the query image feature.

\section{DISCUSSION AND CONCLUSION}

Image retrieval precisions observed in the reported experimental validation illustrate the potential advantages of projecting the ANN characterization values on the membership function, to enhance retrieval performance when a fuzzy similarity is applied. Coding the ANN VT characterization, the nominal distance retrieval correctly identifies the three images that belong to the query image diagnostic class without discriminating their similarity order of importance, but assigns the same relatively higher distance to the other two images which vary in different ways from the query image. Using directly the descriptor vector without the ANN output, the Euclidean distance identifies two of the images, $I_{1}$ and $I_{2}$, which belong to the query image diagnostic class, as being closer to it than $I_{5}$, whereas $\mathrm{I}_{3}$ and $\mathrm{I}_{4}$, have different distances. Regardless of the properly ordered image sequence, a distance threshold should be fixed, to decide whether $I_{5}$ or $I_{3}$ belong to the query image diagnostic class.

The proposed approach on the other hand, measures the fuzzy similarity between the fuzzified ANNs characterization, taking into account their uncertainty, while allowing, if required, emphasizing any particular retrieval feature. Thus, depending on the assigned feature importance, even if in some cases the distance threshold issue is unavoidable, the retrieved objects are properly identified, significantly improving the results precision. Such a retrieval behavior is essential to achieve, multiple image and electronic patient record feature correlation in a data mining framework, leading to the extraction of valuable unknown associations.

It is important to note that image indexing performance depends mainly on the ANN training robustness, which must be confirmed by the medical specialist and necessitates a considerable learning database. Notwithstanding this fact, the supervised indexing procedure is flexible, given that different spatial domain descriptors can be applied, as long as the compatible ANN is used for the VT characterization. For that reason, independently of the characterization method, the fuzzy retrieval can be accomplished preserving its accuracy, without results variability. Moreover, the medical specialist is allowed to adjust the retrieval objective using an expertise-based criterion, which is translated by the fuzzy similarity weights.

\section{REFERENCES}

[1] P.H. Lim, R. Debon, B. Solaiman, L. Bressollette, B. Guias, C. Roux, H.T. Chuah, "Toward Determination of Venous Thrombosis Ages by Using Fuzzy Logic and Supervised Bayes Classification," Proc. $23^{\text {rd }}$ International Conference of IEEE EMBS, 2001, 4, pp. 3831-3834.

[2] A. Dahabiah, J. Puentes, B. Guias, L. Bressollette, B. Solaiman, "Comparative Neural Network Based Venous Thrombosis Echogenicity and Echostructure Characterization using Ultrasound Images, ” IEEE Inf. and Com. Technologies, 2006. pp. 992-997.

[3] L. Daniel, L’Echographie Générale en Réanimation, Springer-Verlag, France, 2002, $256 \mathrm{pp}$.

[4] T. Acharya, A.K. Ray, Image Processing: Principles and Applications, Wiley Interscience, 2005, pp. 227-252.

[5] B. Solaiman, R. Lepage, Les Réseaux de Neurones Artificiels et leurs Applications en Imagerie et en Vision par Ordinateur, Université de Québec, Ecole de Technologie Supérieure, 2003, pp. 137-444.

[6] A. Mojsilovic, D.M. Rackov, "On the Selection of an Optimal Wavelet Basis for Texture Characterization," IEEE Trans. Image Processing, 9 (12), 2000, pp. 2043-2050.

[7] D. Marquardt, "An Algorithm for Least-Squares Estimation of Nonlinear Parameters," SIAM J. Appl. Math., 1963, 11, pp. 431-441.

[8] L. Zhang. "Multimedia Information Retrieval Using Fuzzy Semantic Distance," Proc. IEEE International Conference on Natural Language Processing and Knowledge Engineering, 2003, pp. 374-379. 